\title{
EL PENSAMIENTO ECONÓMICO-ESTÉTICO DE SPINOZA PARA PENSAR LA COMUNIDAD
}

Ana María Ayala Román ${ }^{1}$

1 Candidata a Doctora en Filosofía de la Pontificia Universidad Católica de Valparaíso. Becaria CONICYT-PCHA/Doctorado Nacional/2014-63140076. Licenciada y Magíster en Filosofía de la Universidad del Valle (Colombia).

anamariaayala@gmai.com 


\section{RESUMEN}

La Época Moderna inaugura un tipo de pensar fundamentado en un sujeto, titular del pensamiento, y de un cuerpo; esta característica del sujeto va a ser crucial para pensar la construcción del cuerpo político o comunidad, puesto que el individualismo relacionado con esta concepción propone la constitución y permanencia de una comunidad solo cuando existe un orden impuesto por la ley, ley que viene a ser trascendente o soberana. Este es el caso de Hobbes, para quien la institución de la comunidad se da en la inmanencia del derecho natural, pero solo se conserva cuando se erige un poder trascendental. Esta investigación tiene como objetivo reflexionar, desde el pensamiento de Baruch Spinoza, la posibilidad de la existencia de una comunidad instituida desde la inmanencia y flujo de los afectos (economía-estética), y no desde una soberanía trascendente. Para tal objetivo se analizará el pensamiento ontológico-político del pensador holandés, a la vez de lecturas contemporáneas sobre los tópicos centrales de la investigación (estética política, comunidad, afectos, etc.).

\section{INTRODUCCIÓN}

A finales de la época de los 70 Michel Foucault realiza un viraje con respecto al proyecto que tenía cuando escribió Historia de la sexualidad: la voluntad de saber (2001). Este nuevo proyecto lo acercó a estudiar textos antiguos, tanto de griegos como de latinos.

Debía escoger: o bien mantener un plan establecido, acompañándolo de un rápido examen histórico de dicho tema del deseo, o bien reorganizar todo el estudio alrededor de la lenta formación, en la Antigüedad, de una hermenéutica de sí. Opté por este último partido. (Foucault, 2001, p.10).

Este nuevo proyecto foucaultiano se enfoca en las formas au- 
tónomas de construcción del sujeto, es decir, de prácticas de liberación, caracterizadas por la desnormalización del sujeto frente a las prácticas de sujeción. Las prácticas de liberación están relacionadas con una elaboración de la propia vida, el sujeto realiza una "obra de arte personal". El estudio de los clásicos griegos y romanos le permite a Foucault mostrar la existencia de prácticas donde se afirma la propia libertad, donde se busca dar con una cierta ética que no se instituya desde prohibiciones universales. Esta ética pretende dar una cierta forma a la propia vida. Ahora, si bien estas formas de elaboración de la propia vida implican una salida política a la dominación en las sociedades disciplinarias y de control, no es clara en Foucault la implicación de la comunidad en esta propuesta, por lo que podríamos considerar que el filósofo solo elabora una salida individual. Con esto no se está diciendo que Foucault haya caído en un pensamiento de corte liberal, donde lo importante es el individuo, solo se está afirmando el problema de establecer una solución a la liberación en términos de comunidad, y que, a su vez, esta idea para pensar la comunidad no se funde desde la idea de representación. Una respuesta posible a este problema, o tal vez el olvido en la obra de Foucault de este carácter de comunidad para caracterizar la liberación del sujeto de la sujeción, puede ser explicado por la temprana muerte del filósofo, que truncó el plan reciente de su filosofía.

El problema anteriormente enunciado ha sido objeto de diversas lecturas y aproximaciones desde teorías filosóficas contemporáneas. El objetivo de esta investigación pretende otorgar, desde la recepción contemporánea de la obra de Baruch Spinoza, una aproximación a este problema, en particular a partir de las lecturas sobre la obra de Spinoza de Gilles Deleuze, Felix Guattari y Antonio Negri.

Ahora, ¿cómo permite Spinoza dar cuenta de la liberación de 
la sujeción desde una perspectiva comunitaria y no individual? Debe tenerse en cuenta que no existe una distinción entre lo político y lo ético en el pensamiento spinocista, puesto que es en la Ética donde se encuentran las bases de la formación de la comunidad política y el desarrollo de su pensamiento acerca de la servidumbre y libertad humana.

La verdadera política de Spinoza es su metafísica. Contra las potencialidades de ésta se descargan la polémica del pensamiento burgués y las tentativas de mistificación que discurren bajo la sigla "spinozismo". Pero la metafísica spinozista se articula como discurso político, y en este campo desarrolla específicamente algunas de sus potencialidades. Aquí debemos intentar identificarlas. (Negri, 1993, p.357)

La metafísica spinocista, mostrada de manera magistral en la Ética, trata sobre la producción: la producción de la realidad por parte de la potencia de Dios, producción que se muestra como necesaria (no dependiente de la voluntad o pensamiento de Dios, sino de su naturaleza, de su potencia de producir). Esta producción puede verse en los modos finitos, cuerpo y pensamiento (hombre), donde estos son definidos tanto por su constitución (ideas y cuerpos simples) y su poder afectivo.

En efecto, es la producción de afectos el nodo del pensamiento político de Spinoza, pues es en esta producción donde se establece la relación de unos cuerpos con otros, lo que permite dar cuenta del entramado que conforma la comunidad. Esta forma de definir los modos finitos que instala Spinoza permite otra posibilidad para pensar al hombre, no como sujeto-cartesiano: consciente de sí y propietario de un cuerpo, sino desde su capacidad de ser afectado y, por tanto la singularización de los modos finitos pasa no solo por la manera como están constituidos, sino por la relación que establece con otros (relaciones entre inten- 
sidades), dándose así una singularización que siempre compromete otro cuerpo, otro pensamiento².

[...] Todo lector de Spinoza sabe que los cuerpos y las almas no son para él ni substancias ni sujetos, sino modos. No obstante, si uno se conforma con pensarlo teóricamente no ha hecho lo suficiente. Pues, en concreto, un modo es una relación compleja de velocidad y de lentitud en el cuerpo, pero también en el pensamiento, y es un poder de afectar y de ser afectado, del cuerpo o del pensamiento. [...] Concretamente, si se definen los cuerpos y los pensamientos como poderes de afectar y de ser afectados, muchas cosas deberían cambiar. Se definiría un animal o a un hombre no por su forma ni tampoco como un sujeto; se lo definiría por los afectos de los que es capaz. (Deleuze, 2009, p.151)

Por lo dicho anteriormente, es posible establecer que Spinoza entrega una manera singular de pensar la comunidad, como relaciones de flujos de intensidades, esto es de potencias (conatus), permitiendo, a partir de ello, una perspectiva distinta para el desarrollo de la vida humana. Sin embargo, para poder establecer todas las implicancias políticas del giro que realiza Spinoza, en relación con la construcción de la comunidad, es necesario elaborar desde su propio pensamiento una "economía-estética" que permita comprender las implicancias de este horizonte.

Esta propuesta de trabajo se enmarca en un plano de orden postmetafísico o no representativo ${ }^{3}$. Ahora bien, hablar de la co-

2 Es necesario destacar en este punto la posibilidad el quiebre de Spinoza con respecto al proyecto que se puede dilucidar en el pensamiento occidental desde Tomás de Aquino y pasando por su contemporáneo Descartes. Tal pensamiento se funda en una concepción particular de Dios (como ente voluntarioso y sapiente, acto puro), de los entes (como entidades contingentes y dependientes de la voluntad de Dios) y el hombre (entidad contingente pero privilegiada, ya que encarna el atributo del pensamiento).

3 El pensamiento metafísico de la Época Moderna, así como lo vislumbró Heidegger, está orientado por una separación entre sujeto/objeto, esta separación se funda en una concepción del hombre como ego en cuya certeza se instituye lo otro, es decir el objeto. En un pensamiento como este, el pensar sólo puede ser representacional y la verdad es vista como certeza. Sobre la base del pensamiento representacional, la comunidad es sólo vista como suma de individuos, es decir, de egos. 
munidad nos lleva a pensar "lo común", que Spinoza no pone en una identidad (raza, pueblo, territorio, idea), sino más bien en aquello que nos hace singulares: la potencia de ser afectados y de afectar, que en cada cuerpo y pensamiento es diferente. La diferencia que se da en los cuerpos y los pensamientos se encuentra determinada por la forma de expresión de la potencia de Dios en cada uno, además del modo como está compuesto un cuerpo. En este sentido, podemos encontrar la razón por la que Spinoza afirma que una misma cosa puede afectar de diversas formas a diferentes cuerpos (Cfr. Spinoza, 2009, pp.183186). En relación con lo anterior, podemos ver en Spinoza: 1) Un pensamiento que se alza sobre lo común pero que no niega las diferencias, y con esto, los conflictos que son inherentes a todo pluralismo (a este respecto podemos ver una cercanía de Jacques Rancière, puesto que ahí donde hay conflicto hay política). 2) Un pensamiento que piensa lo político y, con ello a la comunidad, como producción desde la potencia humana y no como una representación. Recordemos que para Spinoza, a diferencia del pensamiento representacional de Descartes, las ideas no son representaciones que un yo se forma de otro-objeto, sino que la idea es una forma de expresión de la potencia de pensar. Dicho de otra manera, el pensamiento no es propiedad de un sujeto, sino una forma de expresión de una potencia común.

Ver la política como potencia del hombre y a la comunidad como un resultado de la producción de afectos, sugiere encargarse de varios problemas de índole económico y estético (no representativo), pues como se dijo anteriormente, si la potencia del hombre se entiende como el poder de afectar y ser afectado, y esta afectación no se encuentra dentro del plano representacional (donde hay una separación entre sujeto/objeto) sino dentro de un plano estético (en la inmanencia del cuerpo), la política 
de Spinoza deberá encargarse de la organización de dicha afectación (economía), para de esta forma elevar el poder de acción, que es lo mismo que elevar el conatus.

La propuesta de este proyecto es acercarse a la elaboración de un concepto de "economía-estética" donde se piense a la economía como organización de los flujos de potencias. Esta organización tiene dos aspectos: 1) Por un lado, pensar el límite del cuerpo político o comunidad que se forma, pues Spinoza es claro en afirmar que toda potencia tiene sus límites, puesto que si no existiera se llegaría a la muerte. Este límite está fijado por el conatus tanto, del modo finito como de la comunidad, en tanto que la muerte aparece cuando se da una relación contraria a la fuerza afectiva (Cfr. Spinoza, 2009, pp.211-212). Además, este aspecto nos permite realizar una crítica al pensamiento político tradicional que tiene como base antropológica pensar un individualismo a ultranza, y con ello, el deseo ilimitado de los individuos. 2) Por otro lado, una economía pensada como organización de intensidades de afectos sugiere una dimensión estética, puesto que podemos vincularla al tema de la felicidad y con ello decir que la economía que se generaría en el pensamiento de Spinoza, es una economía que organiza las potencias para producir una vida bella, es decir, feliz (en tanto que lo bello y lo feliz es para Spinoza maneras de afectación positivas, pues aumentan la potencia de acción). La estética de Spinoza tendrá como plano al cuerpo, pues es en este donde se dan los afectos, así podemos entender por qué Spinoza no conceptualiza términos como "belleza", "fealdad", "arte", pues esta conceptualización solo se puede hacer cuando la experiencia estética deviene otra cosa, en este caso, deviene concepto y, es en esta conceptualización donde lo que en un primer momento era inmanente ahora es una trascendencia que funciona como ley (imperativo estéti- 
co). La estética spinocista se da en la inmanencia, en un plano de inmanencia que es el cuerpo (siguiendo a Deleuze). Es en este plano de inmanencia donde las afecciones se producen y con ellas la belleza y la fealdad. En relación con el primer punto, podemos señalar que la construcción de una lectura "económico-estética" de la obra de Spinoza, responde a una nueva aproximación a la obra del autor.

En efecto, la pregunta que guiará esta investigación consiste en dilucidar: ¿Cómo el pensamiento económico-estético de Spinoza ayuda a pensar una posibilidad de comunidad feliz, esto es, activa y libre?

Para desarrollar esta investigación se abordará el problema a partir de tres objetivos específicos, vinculados cada uno de ellos al desarrollo de cada uno de los tres capítulos de la investigación, de modo que la conclusión dé cuenta del objetivo general de la investigación, el que consiste en la construcción de una interpretación económica-estética para el pensamiento político de Baruch Spinoza.

\section{Objetivo general}

Construir una interpretación "económico-estética" del pensamiento spinocista que exprese desde el modo político de su pensamiento el cruce de los conceptos de "comunidad" y "felicidad" como contrapartida a la soberanía trascendente.

\section{Objetivos específicos}

- Contraponer la crítica spinocista al proyecto de subjetividad trascendente en pro de un pensamiento inmanente sobre la vida humana.

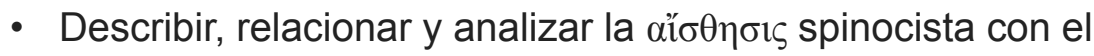


pensamiento inmanente, es decir, sobre el cuerpo.

- Describir y analizar la relación entre economía de los afectos, comunidad y felicidad.

\section{MARCO TEÓRICO}

A la fecha, las lecturas más reconocidas en relación con la interpretación de la obra de Spinoza en claves políticas se podrían categorizar del siguiente modo:

1. Rescate de las pasiones como hecho fundamental en la explicación de la conformación política. Esta lectura destaca el naturalismo spinocista, así como la concepción del hombre como potencia (deseo). Un rasgo fundamental de este tipo de interpretación es la de centrarse en el estudio de la Ética y la analítica de las pasiones realizada por Spinoza, además de señalar la importancia de la desvalorización de las pasiones tristes. Dentro de esta interpretación encontramos a autores como: Robert J. McShea (1968) quien afirma que la razón es una pasión, la finalidad del hombre, como criatura racional, es conservarse a sí mismo a través del entendimiento. Mercedes Allende Salazar Olaso (1988) realiza un estudio exhaustivo de la Ética donde analiza la imaginación, el conocimiento verdadero, el cuerpo y las pasiones. Gregorio G. Kamisnky (1998) resalta el develamiento que realiza Spinoza de los mecanismos y procedimientos político-imaginarios, la superstición y las ilusiones, pues tienen su origen en las pasiones tristes. Diego Tatián (2002) hace un análisis de la positividad de las pasiones en la conformación política. Inmaculada Hoyos Sánchez (2012) destaca el elemento dinámico y materialista del naturalismo spinocista, así como la identificación de la ontología de Spinoza con una ontología de las pasiones. Fernan- 
do A. Bahr (2005), efectúa un examen de las pasiones del miedo y la esperanza y la rehabilitación de ésta última en relación con el concepto de multitud en el Tratado Político.

2. Spinoza como contractualista. Esta lectura sobre la política de Spinoza hace énfasis en conceptos utilizados por Spinoza, tales como: "derecho natural" y "contrato social". Cabe aclarar que la mayoría de estas lecturas intentan realizar un vínculo del pensamiento de Spinoza con el de Thomas Hobbes. A la vez, existen interpretaciones que ven a Spinoza como el precursor del pensamiento de Rousseau. Aunque estos vínculos a veces prestan atención a las diferencias entre los pensadores, siguen manteniendo una noción contractual del pensamiento spinocista. Esta lectura puede encontrarse en los siguientes autores: Walter Eckstein (1944), quien afirma que Rousseau traslada el concepto ético de autonomía a la esfera política y esta libertad ética fue un concepto fundamental en la ética spinocista. J. W. Gough (1967) asevera que la teoría de la obligación política de Spinoza se corresponde estrechamente con la de Hobbes; tal correspondencia se encuentra en la concepción spinocista del derecho natural. Lucien Mugnier-Pollet (1976) reflexiona sobre el pensamiento político de Spinoza centrado en conceptos como: "ley natural", "estado de la naturaleza", "contrato", "contrato social”, "derecho", "paz", "seguridad", "poder absoluto", "relaciones internacionales", y "libertad de pensamiento". F. Tinland (1985) construye una relación entre democracia y legitimidad política. Leiser Madanes (2001) expone la herencia de Descartes y Hobbes en el pensamiento de Spinoza y la radicalización que hace este último de los primeros. W. Bartuschat (2012) realiza un análisis sobre el derecho natural, poder indivi- 
dual y las condiciones de conservación del Estado. Luis Salazar (2002) elabora una comparación entre la teoría de la obligación política de Hobbes y la perspectiva realista de los procesos políticos de legitimación en el pensamiento de Spinoza. W. R. Daros (2008) reflexiona sobre el concepto de "derecho" en relación con el concepto de "poder natural", además de análizar el origen del pacto social centrado en la utilidad común. José García Leal (1982), al igual que Gough, comparte la correspondencia entre Hobbes y Spinoza en lo que respecta al derecho natural, pero presta particular interés en las diferencias sobre el contrato social y las partes que participan de este. André Campos (2009) afirma que el pensamiento político de Spinoza no se opone estrictamente al pensamiento contractualista, sino que intenta corregirlo y darle un nuevo significado desde su interior; Spinoza reconstruiría conceptos contractualistas como los de "naturaleza", "Dios", "individuo", "ley natural", "poder", o "Ley". Ricardo Hurtado Simo (2008) construye un camino común entre Rousseau y Spinoza que se encontraría en el pensamiento sobre la democracia.

3. Relación entre Maquiavelo y Spinoza. Este tipo de interpretaciones se enfocan principalmente en el realismo político de los dos pensadores, y, como consecuencia, la crítica a las utopías. Se resalta la lectura e influencia del florentino sobre el pensador holandés, sobre todo en la crítica a la tradición filosófica y la religión. Entre los intérpretes de Spinoza en esta línea, encontramos a: Louis Althusser (2008), quien sostiene que en los dos filósofos se afirma la vía real del materialismo. Carla Gallicet Calvetti (1972), sostiene que la condición natural del hombre descrita por Spinoza es próxima a la de Maquiavelo, por ello guardan una afi- 
nidad en su pensamiento político. Carlo Altini (1972), declara que los dos pensadores se enfocan en la pacífica estabilidad de las formas del poder político y la impotencia humana como causa de la inestabilidad de las formas políticas. Humberto Schettino (2002), analiza la noción de "imperio" como concepto fundamental en el pensamiento político de los dos autores; sostiene que es posible encontrar un mismo modelo teórico en los dos autores que se denomina: "realismo sofisticado". Steven C. Smith (2007) asegura que, al igual que Maquiavelo, Spinoza asume una actitud realista hacia las pasiones y trata de diseñar una realidad política para los hombres que no pretende ser perfecta. Filippo Lucchese (2011) hace una lectura guiada por las interpretaciones de Althusser y Negri sobre Spinoza, resalta la innovación en el pensamiento de Maquiavelo y Spinoza en la reinvención de los sentidos de conceptos para describir la realidad. Jaime Rubio Angulo (1999) asevera que Spinoza descubre en Maquiavelo la advertencia a los ciudadanos de que las instituciones políticas y los procedimientos políticos, de quienes gobiernan en un miedo constante a la población, son peligrosos para la libertad de todos. Juan Manuel Forte (2009) apunta que en Maquiavelo y Spinoza se dan elementos de cierta tradición republicana, puesto que es esta tradición la que intenta suprimir o minimizar la dominación.

4. Interpretación de "plebe", "vulgo" y "multitudo". Esta interpretación del pensamiento de Spinoza consiste en tomar conceptos que aparecen en el Tratado Teológico-Político y el Tratado Político. Con tales conceptos se pretende abordar temas como el poder popular y constituyente. Además de lo anterior, se pretende recuperar estos conceptos en 
oposición al de "pueblo". Dentro de los exponentes de esta interpretación se encuentran: Antonio Negri y Michel Hardt (2004), quienes proponen a la multitud como multiplicidad irreducible a un uno -conjunto diferenciado-, la multitud es un concepto de clase - clase de las singularidades productivas. La multitud es la potencia ontológica -dispositivo que desea recrear el mundo-. Marilena Chaui (1996, 2004) construye una relación entre multitud y democracia; hace un análisis del concepto "vulgo" en el Tratado Teológico Político como constitutivo del campo político. Paolo Virno (2003) realiza el análisis del concepto de "multitud" en las sociedades postfordistas. Afirma que la "multitud" es contraria a "pueblo"; la multitud es un conjunto de singularidades que actúan concertadamente en la esfera pública. Maurizio Lazzarato (2006) realiza un cambio del concepto "clase" por el de "multitud", este análisis se enfoca en la relación entre multitud y cooperación en una producción inmaterial -producción de afectos, creencias y deseos.

5. Interpretación del pensamiento de Spinoza desde el giro afectivo. Esta interpretación está relacionada con la interpretación que realiza Gilles Deleuze (1975 \& 2009) del pensamiento de Spinoza. Dicha interpretación busca destacar el plano del cuerpo en su poder de afectar y ser afectado, y con ello, la producción de afectos se vuelve central en el pensamiento político, tanto de Spinoza como el de Deleuze. Los siguientes autores están esta línea de interpretación: Brian Massumi (2002) renueva el empirismo radical y la teoría de la percepción de Bergson a través de la filosofía de Deleuze, Guattari y Foucault. Con lo anterior busca relacionar una lógica cultural de la variación de cuestiones como el movimiento, el afecto y la sensación. 
Además, vincula su teoría con medios culturales como la arquitectura, el arte digital y el cine. Rosi Braidotti (2006) propone el concepto de "sustentabilidad ética" como una forma de constitución de formas de subjetividad que pasan por la afectividad, fuerzas e inter-relacionalidad. La pregunta central del análisis es: ¿qué puede un cuerpo? Alexander Carnera (2012) desarrolla una teoría que conecta el valor de la vida y la producción (autogestión), para tal desarrollo se basa en la teoría afectiva de Deleuze y Guattari (cuerpo sin órganos), en la autogestión se producen valores con base en la construcción de buenos encuentros que son subjetivados a través del cuerpo, a través de las capacidades afectivas.

Este breve recorrido por las interpretaciones sobre el pensamiento político de Spinoza permite ver el autor como un continuador de líneas de pensamiento (como la de Maquiavelo y Hobbes) o como un autor original que permite destacar aspectos innovadores y por tanto, posibilidades de repensar lo político. Ahora bien, este recorrido también permite observar que en ninguna de ellas existe un acercamiento explícito al pensamiento económico-estético del autor. De ahí que sea importante rescatar esta dimensión de su pensamiento para pensar problemas políticos actuales, desde coordenadas distintas a la modernidad más clásica. Cabe señalar, que dentro de las líneas interpretativas esbozadas anteriormente, esta investigación considera retomar, de manera crítica, aquellas que ven las pasiones como hecho fundamental de la conformación política, esto porque las pasiones o afectos son de vital importancia en el pensamiento de Spinoza y permiten desarrollar el aspecto estético de esta investigación. También se tendrá en cuenta la línea interpretati- 
va, donde el concepto de "multitudo" es central, puesto que este concepto permite analizar la posición spinocista donde la política no nace del individuo, sino desde las singularidades que no pueden ser pensadas sino en relación, estableciendo a partir de ello una lectura política que rompe con la idea moderna del individuo como eslabón último del aspecto político-social. Por último, es fundamental para nuestro trabajo de investigación, la línea interpretativa inaugurada por Deleuze, pues la teoría de los afectos es de gran importancia para elaborar el concepto de economía-política que queremos construir aquí, puesto que tiene relación con la organización de los afectos para así lograr una comunidad feliz.

\section{MARCO METODOLÓGICO}

El desarrollo de esta tesis tendrá como eje central la lectura y análisis de textos spinocistas que tienen relación directa con los problemas y objetivos propuestos anteriormente. Es necesario resaltar que la investigación propuesta tiene como objetivo elaborar un concepto de economía-estética para interpretar el pensamiento spinociano sobre la comunidad, resaltando la relación de la potencia política de la comunidad con la felicidad, y contraponiendo el pensamiento spinocista con el de una soberanía trascendente (por fuera de la potencia de la comunidad), por tanto la investigación se inscribe en una metodología hermenéutica-constructiva, en tanto se pretende no solo realizar una exégesis interpretativa del pensador holandés, sino construir una categoría con la cual leer su pensamiento. Este proceso constructivo permite leer de manera crítica las diversas interpretaciones que se han hecho sobre la filosofía spinocista, por lo que permite una lectura propia y novedosa. Las etapas que se han formulado para esta investigación son las siguientes: 


\section{SELECCIÓN, CLASIFICACIÓN Y REVISIÓN DE OBRAS}

La investigación se centrará en los libros: Ética demostrada según el orden geométrico, Tratado teológico-político (1976) y Tratado político (2010). Estas obras serán trabajadas en su idioma original para realizar de manera más rigurosa la interpretación de los diversos conceptos y analizar las diversas acepciones, en una primera etapa considerando las diferencias de interpretación desde el propio autor, y en una segunda etapa (segundo momento metodológico) permitirá confrontar las diversas interpretaciones que autores contemporáneos otorgan a los términos presentes en la obra de Spinoza.

En un segundo momento se seleccionarán textos específicos que permiten analizar la crítica spinocista al proyecto de subjetividad trascendente. Para hacer tal selección se tendrá en cuenta la crítica explícita de Spinoza a la filosofía cartesiana, por tanto se trabajarán textos de René Descartes y, para analizar más a fondo esta crítica, se remontará este problema a Tomás de Aquino (del que Descartes es un heredero filosófico) y Averroes (cuyo pensamiento resuena en Spinoza).

En un tercer momento se seleccionarán, clasificarán y revisarán las obras de autores contemporáneos (sobre todo, franceses e italianos, tales como: Michel Foucault, Gilles Deleuze, Felix Guattari, Étienne Balibar, Jacques Rancière, Jean-Luc Nancy, Giorgio Agamben, Antonio Negri y Roberto Esposito) para vincular el pensamiento spinocista a problemas políticos actuales, distinguiendo entre los autores contemporáneos que recepcionan la obra de Spinoza, y aquello que aportan a la investigación desde el contexto para los problemas abordados. En este último punto se tendrá como principal criterio de selección los temas que en esta investigación son centrales: comunidad, soberanía, 
biopolítica, biopoder, multitudo, cuerpo, relación entre estética y política.

\section{CLASIFICACIÓN Y ANÁLISIS CRÍTICO DE CONCEPTOS}

Para desarrollar esta fase se tendrá en cuenta dónde y cómo son definidos los conceptos. Los conceptos que se trabajarán son: persona, soberanía, orden, sujeto, pensamiento, potencia, afectos (cap. 1). Cuerpo, inmanencia, pasiones, deseo, control, imaginación (cap. 2). Afectos, poder político, amor, cuerpo político, multitudo, democracia, comunidad, felicidad (cap. 3).

Después de este registro de conceptos se pasará a establecer relaciones entre los mismos, para de esta forma develar posibles maneras de entenderlos. Recuérdese que este análisis estará haciendo énfasis en el concepto de economía-estética. Para ello la interpretación se construirá a partir de la crítica de estos conceptos, y se articulará desde un análisis deconstructivo que permita la formación de nuevas formulaciones desde el interior de cada concepto. En otras palabras, la formulación crítica tendrá siempre presente la delimitación de lo político-moderno para -desde sus propias determinaciones- abrir nuevos espacios de pensamiento político. Para esto se tendrá en cuenta: 1) procesos históricos en los que se ha construido el concepto, 2) los diversos usos y acepciones del concepto, 3) mostrar la relación y diferencia entre los conceptos, 4) describir las estructuras de pensamiento que se encuentran tras los conceptos.

Este proceso crítico-deconstructivista permite realizar un proceso constructivo, pues al develar las estructuras de pensamiento -tras los conceptos- posibilita la construcción de otras. Este es el objetivo de proponer (construir) una categoría de economía-estética en el pensamiento de Spinoza que permita pensar 
la política, la comunidad y la felicidad desde un pensamiento no-representacional.

Cabe señalar que el análisis crítico, además de contemplar los textos descritos anteriormente, estará acompañado de la lectura de artículos de revistas especializadas sobre los diferentes problemas que se abordarán en esta investigación.

\section{CONCLUSIONES}

El pensamiento de Spinoza realiza una superación de la metafísica tomista-cartesiana, en tanto se concibe al hombre, no como un individuo al que le pertenece un cuerpo y un pensamiento (idea apoyada en el dogma de la encarnación), sino como una potencia que afecta y es afectada por los demás. En esta perspectiva, el pensamiento de Spinoza nos posiciona en un terreno que no solo da cuenta del hombre sino, ante todo, en el campo político, pues no se puede hablar de hombre como un ser individual, sino como un ser que necesariamente es, en relación con los otros.

El pensamiento estético de Spinoza se encuentra en el terreno de la inmanencia. A Spinoza no le interesa conceptualizar trascendentalmente sobre la belleza, la fealdad o el arte, es decir, no le interesa hacer definiciones, puesto que comprende lo estético desde la sensibilidad o las afecciones que un cuerpo pueda producir con otros cuerpos. Es aquí donde lo estético se relaciona con la felicidad y la comunidad, pues está lejos de ser una satisfacción personal (al modo cartesiano), sino que la felicidad es una producción que se da en la relación afectiva entre cuerpos. De esta forma, para Spinoza es importante organizar los flujos de efectos y, sobre todo, fijar límites (que no son trascendentes o imperativos morales) para que el conatus aumente $y$, por tanto aumente la capacidad de actuar. 
El pensamiento económico de Spinoza se encuentra en la organización o gobierno de los afectos. Es de importancia recordar que en Spinoza son fundamentales para toda vida humana buena: la libertad, la felicidad, la actividad y estas solo pueden estar ahí donde los afectos estén activos dentro de los límites singulares. El pensamiento económico de Spinoza tendría como objetivo un "gobierno de sí" que sea útil a la consecución de dichas virtudes.

A la pregunta: ¿Cómo el pensamiento económico-estético de Spinoza ayuda a pensar una posibilidad de comunidad feliz, esto es, activa y libre? Se podría afirmar que la construcción de una economía-estética en el pensamiento de Spinoza permitiría pensar una política, desde la comunidad, por fuera de los límites del pensamiento representacional, es decir, posibilitaría pensar una política inmanente para que, de este modo, el poder político hiciera de la vida humana una vida activa, liberada de la servidumbre de los afectos tristes y de quienes los manipulan.

\section{REFERENCIAS BIBLIOGRÁFICAS}

Allende Salazar Olaso, M. (1988). Spinoza, filosofía, pasiones y política. Madrid: Alianza Editorial.

Althusser, L. (2008). La Soledad de Maquiavelo. Marx, Maquiavelo, Spinoza. Madrid: Akal.

Altini, C. (1972). Spinoza, lettore del Machiavelti. Milán: Universitá Cattolica.

Bahr, F. (2005). Spinoza. El miedo, la esperanza y la vida política. en Magíster: Revista Miscelánea de Investigación, 21.

Balibar, É. (2011). Spinoza y la política. Buenos Aires: Prometeo Libros.

Bartuschat, W. (2012). Spinoza über Macht und recht in der politik. En Teoría, 32(2), 153-167. 
Bertrand, M.(1983). Spinoza et l'imaginaire. París: Presses Universitaires de France.

Braidotti, R. (2006). The Ethics of Becoming Imperceptible. En Deleuze and Philosophy. Edinburgh: University Press, pp.133159.

Campos, A. (2009). A endoreconstrução do contrato social em Spinoza. En Conatus: Filosofía de Spinoza, 3(5), 11-25.

Chaui, M. (1996). La plebe y el vulgo en el Tractatus Politicus. En H. Giannini, P. Bonzi \& E. López (editores), Coloquio Internacional Spinoza. Facultad de Filosofía y Humanidades. Universidad de Chile, pp.69-95.

Chaui, M. (2004). Política en Spinoza. Buenos Aires: Gorla.

Carnera, A. (2012). The affective turn: The ambivalence of biopolitics within modern labour and management. En Culture and Organization, 18, 69-84.

Daros, R. (2008). Contrato social, derechos privados y educación en la Modernidad. Rosario: Universidad del Centro Educativo Latinoamericano.

Daros, R. (2008). El poder natural como derecho y el contrato social de racional utilidad en Baruch Spinoza. En Pensamiento: Revista de Investigación e Información filosófica, 64(239), 71-96.

Deleuze, G. (1975). Spinoza y el problema de la expresión. Barcelona: Muchnik Editores.

Deleuze, G. (2009). Spinoza: filosofía práctica. Barcelona: Tusquets Editores.

Eckstein, W. (1944). Rousseau and Spinoza: Their Political Theories and Their Conception of Ethical Freedom. En Journal of the History of Ideas, 5(3), 259-29.

Forte, J. M. (2009). La vis dominandi en la tradición republicana: Maquiavelo y Spinoza. En Res Pública, (21), 85-95.

Foucault, M. (2001). Historia de la sexualidad (tomo 2). México: 
Siglo Veintiuno Editores.

Gallicet Calvetti, C. (1972). Spinoza, lettore del Machiavelti. Milán: Universitá Cattolica.

García Leal, J. (1982). La teoría del contrato social: Spinoza frente a Hobbes. En Revista de Estudios Políticos, 28, 125-194.

Gough, J. W. (1967). The Social Contract. Oxford: Clarendon Press.

Hurtado Simo, R. (2008). Tres visiones sobre la democracia: Spinoza, Rousseau, Tocqueville. En A Parte Rei, 56, 1-22.

Kamisnky, G. (1998). Spinoza: la política de las pasiones. BarceIona: Editorial Gedisa.

Lazzarato, M. (2006). Por una política menor. Acontecimiento y política en las sociedades de control. Madrid: Traficantes de Sueños.

Lucchese, F. (2011). Conflict, Power, and Multitude in Machiavelli and Spinoza: Tumult and Indignation. London-New York: Continuum.

Madanes, L. (2001). El árbitro arbitrario: Hobbes, Spinoza y la libertad de expresión. Argentina: Editorial Universidad de Buenos Aires.

Massumi, B. (2002). Parables for the Virtual: Movement, Affect, Sensation. Duke: University Press.

McShea, R. (1968). The Political Philosophy of Spinoza. Nueva York-Londres: Columbia University Press.

Mugnier-Pollet, L. (1976). La philosophie politique de Spinoza. París: Vrin.

Negri, A. (1993). La anomalía salvaje. Ensayo sobre poder y potencia en B. Spinoza. Barcelona: Anthropos.

Negri, A. \& Hardt, M. (2004). Multitud. Guerra y democracia en la era del Imperio. Buenos Aires: Debate.

Rubio Angulo, J. (1999). Libertad y potencia en Spinoza. En Universitas Philosophica, 32. Recuperado de http://www.javeria- 
na.edu.co/publicaciones/uniphilo/resumen/res_32g.htm

Salazar, L. (2002). El problema de la obligación política en Hobbes y Spinoza. En Diánoia, 47, 67-88.

Sánchez Hoyos, (2012). La ontología naturalista de Spinoza como ontología de la Pasión. En Logos, (45), 95-122.

Schettino, H. (2002). Política e imperium en Maquiavelo y Spinoza. En Diánoia, XLVII, 37-66.

Smith, Steven C. (2007). Spinoza y el libro de la vida. Libertad y redención en la ética. Madrid: Biblioteca Nueva.

Spinoza, B. (1976). Tratado Teológico-Político. Salamanca: Ediciones Sígueme.

Spinoza, B. (2009). Ética demostrada según el orden geométrico. Madrid: Editorial Trotta.

Spinoza, B. (2010). Tratado Político. Madrid: Editorial Alianza.

Tatián, D. (2002). La cautela del salvaje: pasiones y política en Spinoza. Buenos Aires: Adriana Hidalgo Editora.

Tinland, F. (1985). Hobbes, Spinoza, Rousseau et la formation de l'idée de démocratie comme mesure de la légitimité du pouvoir politique. En Revue philosophique de la France et de l'etranger, (2), 195-222.

Virno, P. (2003). Gramática de la Multitud. Para un análisis de las formas de vidas contemporáneas. Madrid: Traficantes de Sueños. 